\title{
STUDIES ON THE SYNTHESIS AND STRUCTURE OF CORDYCEPIN MONOPHOSPHATE
}

\author{
FRITZ ROTTMAN*, MARY LOU IBERSHOF AND ARMAND J. GUARINO** \\ The Department of Biological Chemistry, The University of Michigan, \\ Ann Arbor, Mich. (U.S.A.)
}

(Received May Ist, I963)

\begin{abstract}
SUMMARY
Cordycepin monophosphate has been synthesized by two methods. One of these was chemical and involved treatment of cordycepin with cyanoethyl phosphate in the presence of $N, N^{\prime}$-dicyclohexylcarbodiimide with subsequent alkaline hydrolysis. The other involved a phosphate transfer from uridine $5^{\prime}$-phosphate to cordycepin utilizing an enzyme prepared from fresh wheat sprouts. In each instance, the product isolated proved to be the monophosphate derivative and the position of attachment of the phosphate group was shown to be on the primary hydroxyl group of the sugar.
\end{abstract}

\section{INTRODUCTION}

Cordycepin, a nucleoside produced by the mold Cordyceps militaris, has been shown to inhibit the growth of a number of strains of Bacillus subtilis ${ }^{1}$. In vivo it inhibits the growth of the Ehrlich mouse-ascites tumor ${ }^{2}$, and when incubated with the same cells in vitro has been shown to inhibit the uptake of labeled orthophosphate into nucleic acids $^{3}$. We have been interested in determining the mechanism by which this nucleoside inhibits growth in general. KLENOW ${ }^{3}$ demonstrated the appearance of three new nucleotides in the acid-soluble extract of Ehrlich ascites-tumor cells when these cells were incubated in the presence of cordycepin. These nucleotides were tentatively identified as the mono-, di-, and triphosphate derivatives of cordycepin, but the position of attachment of the phosphate was not ascertained. In the same work Klenow postulated that the inhibiting effect of cordycepin on nucleic acid synthesis might be due to the formation of the triphosphate derivative or a closely related compound.

In studies with Bacillus subtilis ${ }^{4}$, we reached the conclusion that the monophosphate derivative of cordycepin is the compound responsible for growth inhibition of this organism. For these studies we needed a source of cordycepin phosphate of known structure. This report presents two methods used for the synthesis of cordycepin monophosphate; one is enzymatic, while the other is chemical. Since cordycepin contains one primary and one secondary hydroxyl group, we also present

* Present address: National Institutes of Health, Bethesda, Md. (U.S.A.).

** Present address: Department of Biochemistry, Woman's Medical College of Pennsylvania, Philadelphia, Pa. (U.S.A.). 
evidence that the location of the phosphate ester in the compound obtained by either method is on the primary hydroxyl group. The structure of cordycepin phosphate proposed as a result of these studies is indicated in Fig. I.

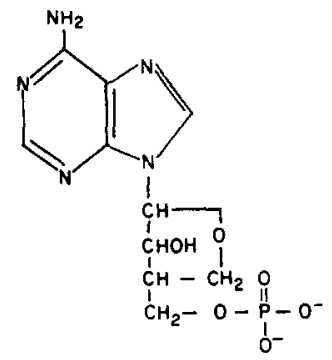

Fig. I. Cordycepin monophosphate.

\section{MATERIALS AND METHODS}

Cordycepin was obtained from culture filtrates of Cordyceps militaris by a published procedure $^{5}$. The compound used in these studies was recrystallized several times from water. Uridine $5^{\prime}$-phosphate was purchased from either Calbiochem or Sigma Chemical Co. Barium cyanoethyl phosphate and $N, N^{\prime}$-dicyclohexylcarbodiimide were purchased from Calbiochem. Yeast adenylic acid was purchased from Schwarz Laboratories. Pure adenosine $2^{\prime}$-phosphate and adenosine $3^{\prime}$-phosphate were obtained from this mixture by adsorption and elution from Dowex-I-Cl ion-exchange columns. Snake venom (Crotalus adamanteus) was purchased from Sigma Chemical Co. and was used without further purification.

Phosphate determinations were carried out by the $\mathrm{KING}^{6}$ modification of the Fiske-Subbarow determination? ${ }^{7}$. Cordycepose was determined by the modified anthrone procedure of KREDICH AND GUARINO ${ }^{\mathbf{5}}$ utilizing cordycepin as a standard and calculating the concentration of cordycepose present from the molecular extinction coefficient for cordycepin.

Periodate oxidation was carried out according to the procedure of MarinetTI AND RousER ${ }^{8}$. Protein concentrations were determined by the method of RoBINSON AND HOGDEN ${ }^{9}$.

\section{RESULTS}

\section{Enzymatic synthesis of cordycepin phosphate}

The procedure used was in essence that of phosphate transfer ${ }^{10}$ from uridine $5^{\prime}$-phosphate to cordycepin. The mixture of nucleosides and nucleotides present at the end of the incubation were separated by ion-exchange chromatography. The enzyme used in these studies was prepared from wheat seedlings by the procedure of BARNER AND COHEN ${ }^{11}$. Wheat was allowed to germinate for 7 days in the dark in the presence of Arasan to prevent mold growth. The fresh sprouts were harvested, washed with water, minced with scissors, and ground in a pre-chilled mortar with $5 \mathrm{ml}$ of water per gram of wet sprouts. The crude homogenate was centrifuged and the precipitate reextracted in the same manner. The supernatant fluids from both extractions were combined and stored at $-20^{\circ}$. This soluble extract was used without further purification and had a protein concentration of apprcx. $0.7 \mathrm{mg} / \mathrm{ml}$. 
In a typical experiment, $264 \mu$ moles of cordycepin and $2530 \mu$ moles of uridine $5^{\prime}$-phosphate were incubated in $0.38 \mathrm{M}$ acetate buffer $\left(\mathrm{pH}_{5}\right)$ in a final volume of $42 \mathrm{ml}$ for $3 \mathrm{~h}$ at $30^{\circ}$. At the end of this period the reaction was stopped by adding trichloroacetic acid (5\% final concentration). After standing for $\mathrm{I} h$ at $24^{\circ}$, the suspension was centrifuged and the supernatant fluid was treated with charcoal $(0.3 \mathrm{mg}$ charcoal per absorbancy unit at $260 \mathrm{~m} \mu$ ). Preliminary evidence that the reaction proceeded as expected was obtained by removal of an aliquot prior to the charcoal treatment and chromatographing it on Whatman No. I filter paper. Four ultravioletabsorbing compounds were visualized, the $R_{F}$ 's of which are given in Table I. Evidence

\section{TABLE I}

CHROMATOGRAPHY OF REACTION PRODUCTS AFTER INCUBATION WITH WHEAT PHOSPHOTRANSFERASE

As described in the text, at the end of a 3 -h incubation period, an aliquot was removed and chromatographed on Whatman No. I filter paper by descending chromatography. The chromatogram was developed in a solvent system containing $0 . \mathrm{I} \mathrm{M}$ phosphate $\mathrm{pH} 6.8$ (I00 ml), ammonium sulfate $(60 \mathrm{~g})$ and $n$-propanol $(2 \mathrm{ml})$, for a period of $\mathbf{I} 8 \mathrm{~h}$ by which time the solvent had migrated off the end of the paper. Compounds were visualized with an ultraviolet lamp. The values given are the distances migrated by the compound from the origin divided by the distance from the origin to the end of the paper in $\mathrm{cm}$.

\begin{tabular}{lcccc}
\hline & & \multicolumn{2}{c}{ Relative migration rate } \\
\hline Deproteinized incubation mixture & 0.10 & 0.20 & $0.5^{8}$ & $0.7 \mathbf{I}$ \\
Uridine & - & - & - & - \\
Uridine 5'-phosphate & - & - & - & 0.73 \\
Cordycepin & 0.08 & - & - & - \\
Adenosine 5'-phosphate & - & 0.24 & - \\
\hline
\end{tabular}

is presented in this table for the existence of the four compounds expected in such a reaction; uridine, uridine $5^{\prime}$-phosphate, unreacted cordycepin and cordycepin phosphate. The latter compound was assumed to be cordycepin phosphate, because it had a migration rate comparable to that of adenosine $5^{\prime}$-phosphate.

The charcoal-treated solution was centrifuged and the supernatant fluid discarded after an absorbancy determination at $260 \mathrm{~m} \mu$ showed that most of the ultravioletabsorbing material had been adsorbed. In most instances, better than $94 \%$ of the ultraviolet-absorbing material was removed from the supernatant fluid by the charcoal treatment.

Elution of the reaction products from charcoal was achieved by resuspending the charcoal in $50 \mathrm{ml}$ of a mixture containing $95 \%$ ethanol-conc. ammonia-water $(5: I: 24)$ per gram of charcoal. This suspension was stirred for $2 \mathrm{~h}$ at $37^{\circ}$ which resulted in the elution of $70 \%$ of the total ultraviolet-absorbing material. The charcoal was removed by centrifugation and the supernatant fluid was concentrated to dryness on a rotary evaporator in vacuo at a bath temperature not exceeding $40^{\circ}$. The dry material was dissolved in I $\mathrm{ml}$ of water per 5000 absorbancy units, and the $\mathrm{pH}$ of the solution adjusted to 7.0-7.2 with $\mathrm{NaOH}$. The ultraviolet-absorbing compounds were adsorbed on a Dowex-I-formate column, $35 \mathrm{~cm}$ high $\times 2 \mathrm{~cm}$ in diameter. The resin column was subsequently washed with water, which removed considerable ultraviolet-absorbing material which was shown to be primarily uridine. These fractions were discarded. The resin was then eluted with o.I $\mathrm{N}$ formic acid which removed approx. Io \% of the total ultraviolet-absorbing material which proved to be cordy- 
cepin phosphate. Under these conditions the uridylic acid was not eluted. The fractions containing the pure compound were pooled and treated with charcoal (I mg per absorbancy unit at $260 \mathrm{~m} \mu$ ). The charcoal was removed by centrifugation and eluted with the ethanol-ammonia-water mixture described above. This elution was essentially quantitative.

The eluate was concentrated to dryness in a rotary evaporator in vacuo at a bath temperature not exceeding $40^{\circ}$. The residue was dissolved in water and the cordycepin phosphate precipitated as the barium salt with alcohol $(75 \%$ final concentration). The sodium salt was prepared by passing a solution of the barium salt through a Dowex-50- $\mathrm{Na}^{+}$resin column and pooling the fractions containing cordycepin phosphate. The final solution was stored at $-20^{\circ}$. The analysis of the compound present in the resulting solution is presented below with that for the chemically synthesized product. In several preparations by the above procedure it was possible to obtain $80 \mu$ moles of the sodium salt which represented an overall yield of approx. $30 \%$.

\section{Chemical synthesis of cordycepin phosphate}

The synthesis and purification of cordycepin phosphate was carried out as described by TENER ${ }^{\mathbf{1 2}}$ for the synthesis of deoxycytidine $5^{\prime}$-phosphate. The method involves the reaction of cordycepin with cyanoethyl phosphate in the presence of $N, N^{\prime}$-dicyclohexylcarbodiimide, followed by alkaline hydrolysis and chromatography of the reaction products on a Dowex-I-formate resin column, X8, 200-400 mesh. Although cordycepin contains two free hydroxyl groups no attempt was made to block the secondary hydroxyl group. Both groups were allowed to react after which the $2^{\prime}$-phosphate derivative was separated from the derivative containing the phosphate on the primary hydroxyl group by use of an anion-exchange resin column.

A column $(\mathrm{I} \mathrm{cm} \times 30 \mathrm{~cm})$ of Dowex- $\mathrm{I}$-formate ion-exchange resin, $\mathrm{X} 8,200-400$ mesh, was used to separate the reaction products. The resin column was first washed with water until the absorbancy of the eluate was less than $0 . I$, and then with o.I M formic acid which resulted in the elution of two fractions containing ultravioletabsorbing compounds. The amount of ultraviolet-absorbing material present in each fraction was approximately equal. The compound eluted in the first peak was presumed to be the $2^{\prime}$-phosphate derivative. Although the substance present in this fraction was not characterized, the properties of adsorbability and subsequent elution from charcoal indicated that the ultraviolet-absorbing substance was a nucleoside, and that probably the phosphate was lost during the isolation procedure with formic acid. The material eluted in the second peak contained an acid-stable phosphate, and was easily adsorbed and eluted from charcoal, which is characteristic of nucleotides. If the column was subsequently treated with 0 .I $\mathrm{M}$ formic acid and $0.5 \mathrm{M}$ ammonium formate, a small amount of ultraviolet-absorbing material (usually one-tenth that present in the other two fractions) was eluted which was presumed to be the diphosphorylated derivative.

The ethanol-ammonia-water eluant of the second ultraviolet-absorbing peak containing the acid-stable phosphate was evaporated to dryness on a rotary evaporator and subsequently dissolved in water. Cordycepin phosphate was precipitated from this solution as the barium salt in $70 \%$ ethanol. Before analysis, the barium salt 
was converted to the sodium salt by passage over Dowex-50- $\mathrm{Na}+$ ion-exchange resin. The overall yield in this synthesis was $15 \%$ calculated on the basis of the starting cordycepin.

\section{Proof of structure of cordycepin phosphate}

Both the enzymatically and chemically synthesized cordycepin phosphate were found to be homogeneous in three solvent systems on paper chromatography. Their absorption spectra both in acid and alkali were characteristic of an N-9 substituted adenine. Both products liberated inorganic phosphate quantitatively when incubated with crude snake-venom phosphatase, while in our hands the same enzyme failed to liberate inorganic phosphate from adenosine $2^{\prime}$-phosphate and adenosine 3 '-phosphate.

The chemical analysis of both the chemically and enzymatically synthesized products are given in Table II. In each case the concentration of adenine was calculated utilizing a molecular extinction coefficient for cordycepin of $1.45 \cdot \mathrm{IO}^{4}$ per mole at $260 \mathrm{~m} \mu$. The micromolar concentrations of the cordycepose and organically bound phosphate were divided by the micromolar concentration observed for adenine to

TABLE II

ChEMICAL ANALYSIS OF CORDYCEPIN PHOSPhate

Analyses were carried out as described in the text.

\begin{tabular}{lccc}
\hline & Mdenine & Cordycepose & $\begin{array}{c}\text { Organic } \\
\text { phosphate }\end{array}$ \\
\hline Chemically synthesized product & & I.I & 0.99 \\
Enzymatically synthesized product & 1.0 & 0.96 & 1.03 \\
Theory & 1.0 & I.0 & I.0 \\
\hline
\end{tabular}

give the molar ratios observed in Table II. Since cordycepose does not give the characteristic anthrone color in the phosphorylated form, the anthrone procedure was carried out after removal of the phosphate by incubation with snake-venom phosphatase.

Periodate oxidation was used to determine the position of the phosphate in each of the synthesized products. Unhydrolyzed cordycepin phosphate, when treated according to the procedure described in the legend of Fig. 2, failed to consume periodate within a 15 -min period. This finding is in agreement with theory because the parent compound does not have a pair of adjacent unsubstituted hydroxyl groups.

On the other hand, if cordycepin monophosphate were hydrolyzed under conditions in which the $N$-glycosidic bond was split the pentose phosphate liberated should consume one mole of periodate if the phosphate ester involved was present on the primary hydroxyl group. Cleavage of the nucleotide was accomplished by heating cordycepin monophosphate with a suspension of Dowex-50- $\mathrm{H}^{+}, \mathrm{X} 8,200-400$ mesh for I $h$ at $100^{\circ}$. The resin, which removed $99 \%$ of the original ultraviolet-absorbing material present in solution, was separated by centrifugation. All of the phosphate originally present was in the supernatant fluid and still organically bound. This observation had the added implication that the phosphate was present on the primary hydroxyl group of the sugar rather than the 2-position because we found that 


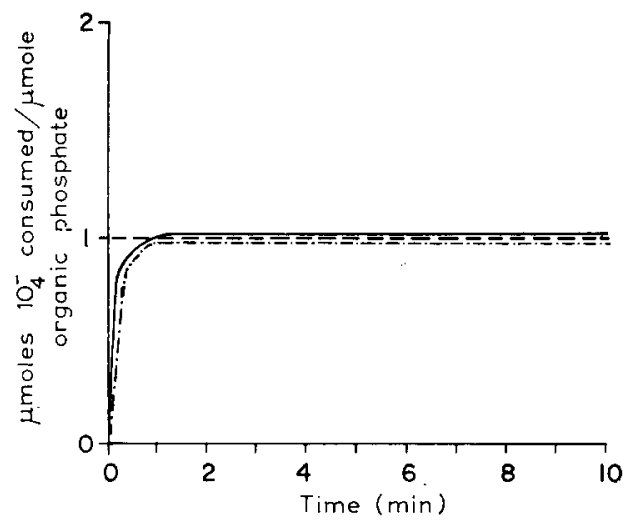

Fig. 2. Periodate consumption by cordycepose phosphate. Reaction vessels contained in a final volume of $1.0 \mathrm{ml}$ : to $\mu$ moles of sodium metaperiodate, $0.08 \mathrm{M}$ acetate buffer $(\mathrm{pH} 5.0$ ) and a suitable aliquot of cordycepose phosphate. The reaction was allowed to proceed at room temperature and was followed spectrophotometrically at $310 \mathrm{~m} \mu$ in a Gilford Multiple Absorbance recorder. The amount of cordycepose phosphate present at the beginning of the experiment was calculated from the concentration of organically bound phosphate. The $\mu$ moles of periodate consumed in the reaction was divided by the $\mu$ moles of organically bound phosphate originally present to give the values present in the graph. - - , theoretical consumption of periodate by cordycepose phosphate; - , cordycepose phosphate obtained from chemically synthesized cordycepin monophosphate; -.-, cordycepose phosphate obtained from enzymatically synthesized cordycepin monophosphate.

similar treatment of adenosine $2^{\prime}$-phosphate liberates significant amounts of inorganic phosphate. The amount of cordycepose phosphate present was calculated from the organically bound phosphate concentration after the Dowex treatment, and an aliquot was taken for oxidation by periodate as described in the legend of Fig. 2 . Within experimental error (Fig. 2), both products consumed the theoretical amount of one mole of periodate after removal of the adenine moiety.

If the phosphate ester of the parent compound had been on the $2^{\prime}$-position, one might have expected a slight but significant liberation of inorganic phosphate as a result of the treatment with Dowex-50- $\mathrm{H}^{+}$resin. As was just mentioned above, this was not observed experimentally. If the phosphate in this position, however, remained stable under the conditions required for cleavage to adenine and pentose phosphate, the latter compound should not have consumed periodate. As shown in Fig. 2, the cordycepose phosphate did consume one mole, which is not in agreement with this position of attachment. The conditions of periodate oxidation could result in a loss of phosphate had this molecule been esterified at the 2-position, but this is not likely since the oxidation was carried out at $\mathrm{pH}_{5}$. In any event, if this were true the product cleaved by treatment with Dowex-50- $\mathrm{H}^{+}$resin would still have consumed a mole of periodate. If this had been the case, however, the phosphate present at the end of the oxidation with periodate should have been totally inorganic in form. In the experiment shown in Fig. 2, aliquots were removed for both an inorganic and total phosphate analysis, and in both the chemically and enzymatically synthesized product all of the phosphate was still organically bound at the end of the oxidation period.

These results leave little doubt that cordycepin monophosphate synthesized by both the enzymatic and chemical routes described above has the structure indicated in Fig. I. 
Another interesting facet of this work pertains to the specificity of the snakevenom phosphatase of Crotalus adamanteus. Although we did not purify this enzyme, we found that it did not liberate inorganic phosphate from adenosine 2 '-phosphate, adenosine 3 -phosphate, ribose 5-phosphate, cordycepose phosphate, or glucose 6-phosphate; but did from adenosine 5'-phosphate and cordycepin phosphate. $\mathrm{KLENOW}^{3}$ also showed that cordycepin monophosphate obtained from the Ehrlich mouse-ascites tumor experiments liberated inorganic phosphate when incubated with the snake-venom enzyme. Presumably, his product also had the phosphate on the primary hydroxyl group. This phosphatase is usually considered specific for $5^{\prime}-$ phosphates of nucleotides, having poor ability to cleave $2^{\prime}$ - or $3^{\prime}$-nucleoside phosphates or hexose 6-phosphates. As a result of our work we might say that this enzyme is specific for nucleotides in which the phosphate is esterified to a primary hydroxyl group. For the common nucleotides containing ribose or deoxyribose, this hydroxyl group is also the $5^{\prime}$-position of the nucleotide. Cordycepin, however, is a nucleoside containing a branched-chain carbohydrate and as such does not have a 5 -position comparable to that of the nucleotides containing ribose or deoxyribose.

\section{ACKNOWLEDGEMENTS}

This work is taken in part from a thesis submitted by F. R. to the Rackham School of Graduate Studies in partial fulfilment for the degree, Doctor of Philosophy.

This work was supported in part by a research grant from the United States Public Health Service (CA-0522I), and by a predoctoral Public Health Service Fellowship to F. R. (No. GF-I7, I08) from the Division of General Medical Sciences, Public Health Service. ,

We wish to thank Mrs. T. Lauderbaugh for some of the analyses.

\section{REFERENCES}

1 K. G. Cunningham, S. A. Hutchinson, W. Manson and F. S. Spring, J. Chem. Soc., (I95I) 2299.

2 D. V. Jagger, N. M. Kredich and A. J. Guarino, Cancer Res., 2 I (I96I) 2 I6.

3 H. Klenow, Biochem. Biophys. Res. Commun., 5 (I96r) I 56.

* F. Rottman and A. J. Guarino, Federation Prog., 22 (I963) 533.

5 N. M. Kredich and A. J. Guarino, Biochim. Biophys. Acta, 41 (I960) 363.

- E. J. KING, Biochem. J., 26 (I932) 292.

7 C. H. Fiske and Y. Subbarow, J. Biol. Chem., 66 (ig25) 375.

8 G. V. Marinetti and G. Rouser, J.Am. Chem. Soc., 77 (I955) 5345.

- H. W. Robinson and C. G. Hogden, J. Biol. Chem., i35 (I940) 707 .

$10 \mathrm{G}$. Brawerman and E. Chargaff, J.Am. Chem. Soc., 75 (I953) 2020.

11 H. D. Barner and S. S. Cohen, J. Biol. Chem., 234 (I959) 2987.

12 G. M. TENer, in M. J. Coon, Biochemical Preparations, Vol. 9, John Wiley and Sons, New York, r962, p. 5 . 